\title{
Fuzzy Neural Network Model for Rules Generating of the Objects State Determining in Uncertainty
}

\author{
${ }^{1}$ Ilyas I. Ismagilov, ${ }^{2}$ Linar A. Molotov, ${ }^{3}$ Alexey S. Katasev, ${ }^{4}$ Lilia Yu. Emaletdinova, ${ }^{5}$ Dina V. Kataseva \\ 1,2 Kazan Federal University \\ ${ }^{3,4,5}$ Kazan National Research Technical University named after A.N. Tupolev \\ Email:fsnugaev@kpfu.ru
}

Received: 02 ${ }^{\text {nd }}$ November 2018, Accepted: $28^{\text {th }}$ November 2018, Published: 31 $^{\text {st }}$ December 2018

\begin{abstract}
This article solves the problem of modelling a fuzzy neural network to generate fuzzy rules and to use them for determining the state of objects in conditions of uncertainty. Traditional methods of mathematical statistics do not allow constructing adequate models of objects under the specified conditions. Therefore, at present, the solution of many problems is based on the use of data mining technologies using the methods of fuzzy logic. The traditional approach to the construction of fuzzy systems is associated with the need to involve an expert to formulate fuzzy rules. To eliminate this drawback, the automation of generating fuzzy rules based on machine learning methods is relevant. One of the approaches to solving this problem is to build a fuzzy neural network and instruction in it based on the data characterizing the object under study. The implementation of this approach required the choice of the type of fuzzy rules, taking into account the peculiarities of the processed data. In addition, the development of an algorithm of logic rule-based inference was required. The stages of the algorithm determine the number of layers in a fuzzy neural network and their functionality. An algorithm for learning a fuzzy neural network has been developed, as a result of which a system of fuzzy-production rules is built. In order to assess the effectiveness of the developed model of a fuzzy neural network, a software package has been implemented. On its basis, the studies on assessment of the classifying ability of the generated rules using the example of data analysis from the UCI Machine Learning Repository have been conducted. The research results show that the classifying ability of fuzzy rules is not inferior to other classification methods in accuracy. In addition, the inferencing algorithm based on the rules makes it possible to successfully perform the classification in the absence of some of the source data. This proves the significance of the obtained results and the effectiveness of the proposed approach for solving the problem of instance state estimate in conditions of fuzziness, heterogeneity and the lack of a part of the analyzed data.
\end{abstract}

\section{Keywords}

Fuzzy Neural Network, Fuzzy-Production Rule, Generation of Fuzzy Rules, Object State Estimate

\author{
Introduction \\ assessing the state of objects, characterized by the following features: \\ - the presence of a large number of inputs, outputs and states; \\ - different types of parameters; \\ - uncertainty, fuzziness and incompleteness of data at the input of the object; \\ - fuzzy severity of the structure.
}

Currently, in various application domains there is a need to solve practical problems associated with modeling and

Traditional approaches based on mathematical statistics [1,2] or simulation modeling [3,4] do not allow building adequate models of objects in the indicated conditions. Therefore, at present, artificial intelligence methods are used when solving many problems related to the assessment of the state of objects [5-10].

The theory of fuzzy sets [11-15], as one of the directions of artificial intelligence, enables to construct fuzzy models of objects. Methods of fuzzy logic are widely used to solve problems of diagnostics, control, support for decision-making in various subject areas [16-19]. However, in spite of the advantages of fuzzy systems, they have a drawback connected with the need to involve a person in order to form the rules of the knowledge base and a task of the membership functions used in them. The most difficult step in building a fuzzy system is the choice of the form and parameters of the membership functions due to the subjectivity of a person's opinion. Moreover, this process requires considerable time and considerable analytical work, which significantly complicates the development of a fuzzy system.

There are adaptive fuzzy models in which the parameters of the membership functions are adjusted automatically in the learning process based on experimental data [20]. This class of fuzzy models is called "soft systems" [21]. Soft approach to the generation of fuzzy rules is based on the use of data of the methods and data mining algorithms. However, when constructing knowledge bases of soft systems and solving specific problems of assessing the state of objects, it becomes necessary to take into account the features of the analyzed data which impose restrictions on the knowledge representation model and the inferencing algorithm. Therefore, to form adequate fuzzy models for assessing the state of objects in uncertainty, the development of a new approach to the generation of fuzzy rules that adequately describe the patterns in the analyzed data is relevant. 


\section{Methods}

The processes of generating fuzzy rules are associated with data analysis by deriving the laws hidden in them. For this purpose, the technology of insight in databases is used. Its use to generate fuzzy rules for assessing the state of objects in uncertainty requires the choice of a type of the model of knowledge representation, as well as intellectual analysis methods taking into account the peculiarities of the data being processed: large volume, heterogeneity, the presence of certainty and fuzziness in data, lack of part of the source data. Taking into account these features, the following type of fuzzy rules has been chosen:

IF $x_{1}=\vec{A}_{1}\left(w_{1}\right)$ И $x_{2}=\overleftrightarrow{A}_{2}\left(w_{2}\right)$ AND $\ldots x_{\mathrm{n}}=\overleftrightarrow{A}_{\mathrm{n}}\left(w_{\mathrm{n}}\right)$ THEN $y=B[C F]$,

where $x_{i}$ - input parameters, $w_{i} \in[0,1]$ - weighting conditions " $x_{i}=\vec{A}_{i}^{\prime \prime}, \vec{A}_{i}=\left\{A_{i}, \widetilde{A}_{i}\right\}, A_{i}-$ clear value of input, $\widetilde{A}_{i}=\left\{x_{i}, \mu_{\widetilde{A}_{i}}\left(x_{i}\right)\right\}$ - fuzzy value of input, $\mu_{\widetilde{A}_{i}}\left(x_{i}\right)$ - membership function, $y$ - output parameter, $B-$ clear value of output, $C F \in[0,1]$ - validity of the rule.

To assess the state of the object based on the rules (1), an inferencing algorithm has been developed. We introduce the designation:

- $R \in[0,1]-$ the degree of triggering the conditional part of the rule:

$R=\min \left(\mu_{\vec{A}_{i}}\left(p_{i}^{*}\right)\right)$,

where $p_{i}^{*}, i=\overline{1, n}-$ known clear values of $n$ input parameters of the rule,

$\mu_{\vec{A}_{i}}\left(p_{i}^{*}\right)$ - the degree of belonging of input values $p_{i}^{*}$ к $\vec{A}_{i}$ :

- for fuzzy input parameters $\mu_{\widetilde{A}_{i}}\left(p_{i}^{*}\right) \in[0,1]$;

- for clear input parameters $\mu_{A_{i}}\left(p_{i}^{*}\right)=\left\{\begin{array}{l}1, \text { if } p_{i^{*}}^{*}=A_{i} \\ 0, \text { if } p_{i} \neq A_{i}\end{array}\right.$;

- if $p_{i}^{*}$ is absent, then $\mu_{\vec{A}_{i}}\left(p_{i}^{*}\right)$ is not calculated and used in (2);

- $T \in[0,1]-$ the weight of the conditional part of the rule:

$T=\frac{\sum_{k n=1}^{n_{k n}} w_{k n}}{\sum_{i=1}^{n} w_{i}}$

where $w_{i}, i=\overline{1, n}-$ the weights of all restrictions of $\vec{A}_{i}$ to the parameters in the rule,

$w_{k n}, k n=\overline{1, n_{k n}}-$ the weights of restrictions of $\vec{A}_{i}$ with certain values;

- $C \in[0,1]$ - integrated assessment of certainty of solving the rule:

$C=R * T * C F$,

where $C F \in[0,1]$ - certainty of the rule.

To fulfil a rule-based logical inference (1) the following algorithm has been developed.

1. Entering the values $p_{i}^{*}$ of input parameters $P_{j}^{i n}$.

2. For each rule Rule $_{r}, r=\overline{1, N}$ the calculation of the degree of functioning its conditions $R_{r}$ according to the formula (2).

3. The formulation of a conflict set involving the rules with the null degree of functioning: $S_{\text {conf }}=\left\{R_{u l e} \mid R_{r} \neq 0\right\}$, $r=\overline{1, N}$.

4. For all rules Rule $_{r} \in S_{\text {conf }}$ the calculation of the weight of the conditional part of the rule $T_{r}$ by the formula (3) and a complex assessment $C_{r}$ by the formula (4). 
5. Resolving the conflict - the choice of a rule with maximum comprehensive assessment of certainty of solving: Rule $_{r}^{*}: \max C_{r}$.

$r:$ Rule $_{r} \in S_{\text {conf }}$

6. Obtaining the value of $p_{j}^{*}$ of an output parameter $p_{j}$ of the chosen rule $R u l e_{r}^{*}$ as the state of an object.

This algorithm enables to select a decision rule with an output value that determines the desired state of the object. Thus, to solve practical problems of assessing the state of objects, it is necessary to generate a system of rules of (1). For this purpose, a model of a fuzzy neural network has been developed [22].

To construct this model, it was necessary to determine its structure and train the network based on the available data characterizing the object under study. The structure of the model is determined by the following characteristics [22]:

1) the number of input parameters of the object (determines the number of neurons in the input layer of the network);

2) the number of values (gradations) of input parameters (determines the number of neurons in the layer of gradation values of input neurons);

3 ) the number of values of the output parameter of the object (determines the number of neurons in the layer of values of the output neuron);

4) the inferencing algorithm on the system of rules of (1) (determines the number of network layers and their functionality).

Figure 1 demonstrates an example of the structure of fuzzy neural network.

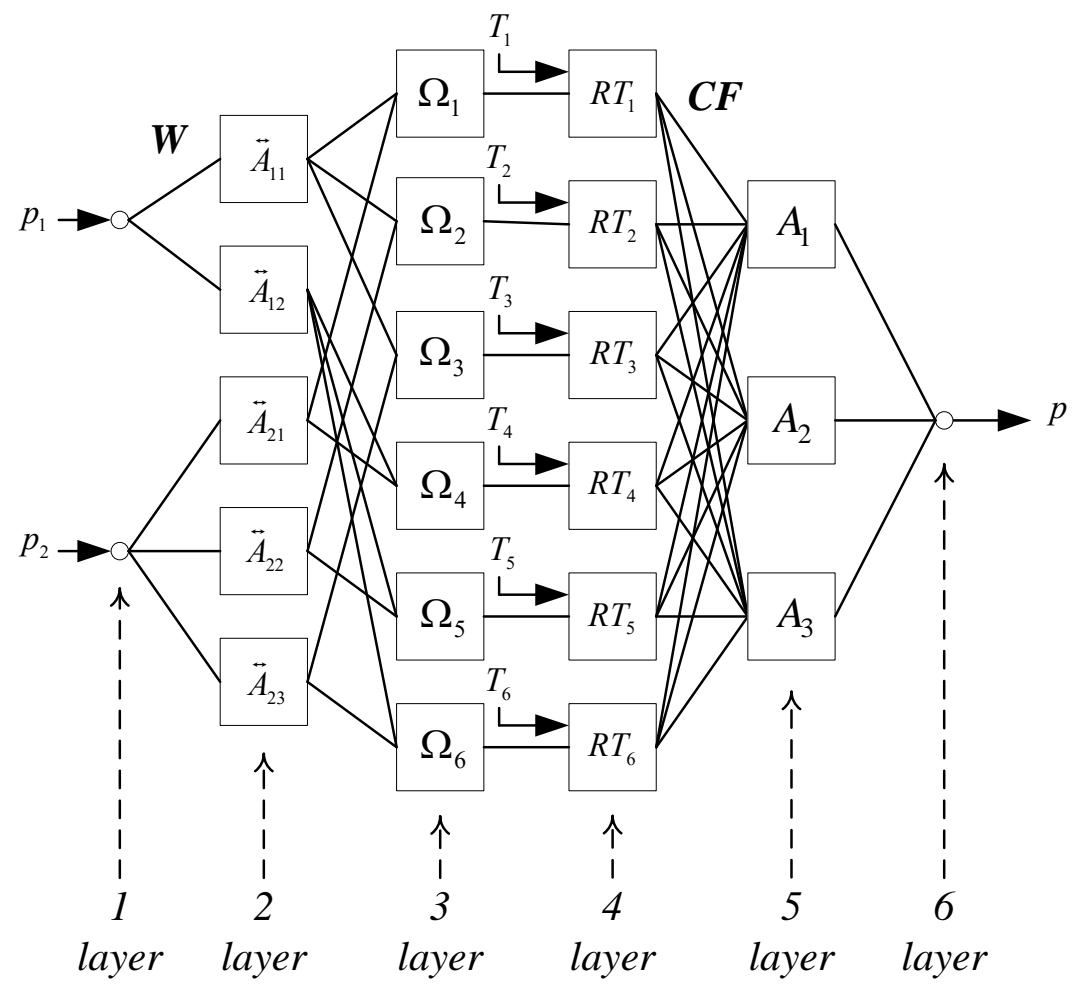

Figure 1: An Example of the Structure of Fuzzy Neural Network

The first layer consists of $P$-neurons which distribute input signals over the neurons of the second layer, consisting of $\vec{A}$ - neurons which model the input conditions of the rules $p=\overleftrightarrow{A}$. The outputs of $\vec{A}$-neurons are the values of membership functions $\mu_{\ddot{A}}\left(p^{*}\right)$. The parameter $w \in[0 . .1]$ which determines the weight of the condition in the rule is associated with each $\vec{A}$-neuron. The third layer consists of $\Omega$-neurons which determine the estimate $R$ of the degree of response of the conditional part of the rules. In the fourth layer the product of the estimates of $R$ and $T$ is calculated. The fifth layer of values of the output neuron consists of $A$-neurons, to the inputs of which the output values of $R T$-neurons and the associated reliability of the $C F$ rules enter. Each $A$-neuron calculates a comprehensive assessment of the reliability of the solution of $C$ and generates the maximum of these estimates at the output. The sixth layer contains a $P$ neuron which forms the value of the output of network $p=A$, corresponding to $A$-neuron with the maximum value of C.

Setting the values of the parameters of the membership functions in the rules is performed by instruction of a fuzzy neural network based on the gradient descent method. In this method, the change in the values of the vector of the 
parameters of membership functions $\mu_{\widetilde{A}_{k}}\left(p_{k}\right)$ for a given input image must be made on the basis of the requirement to minimize the rms error of the output of $\widetilde{A}_{k}^{j}$-neuron:

$\Delta p a r=-\alpha \frac{\partial E_{k}}{\partial p a r}$

where $\quad$ par-the vector of parameters of the membership function $\mu_{\widetilde{A}_{k}}\left(p_{k}\right)=f($ par $)$;

$\alpha \in(0,1]$ - the coefficient of learning rate;

$E_{k}=\frac{\left(d-\hat{\mu}_{\widetilde{A}_{k}}\left(p_{k}^{*}\right)\right)^{2}}{2}-$ the error of output of $\widetilde{A}_{k}^{j}$-neuron;

$\hat{\mu}_{\widetilde{A}_{k}}\left(p_{k}\right)$ - the fragment of continued part of the membership function $\mu_{\widetilde{A}_{k}}\left(p_{k}\right)$, appropriate to the changing parameters;

$d=1$, if it is required to multiply the activity of $A$-neuron;

$d=0$, if it is required to reduce the activity of $A$-neuron.

Thus, the developed algorithm of learning the fuzzy neural network consists of the following steps.

1. To preset a value of learning rate $\alpha \in(0,1]$.

2. To preset an initial value of the counter of classified input images $r e s=0$.

3. To give a recurrent image from the learning sampling of the volume $N$ to the input of network.

4.1. To calculate the output of network for a given input image.

4.2. If the value of the output coincides with the value of the target parameter in input image, to go to step 3 .

4.3. To multiply the value res by 1 .

4.4. To choose two $A$-neurons to change their output activity.

4.5. To determine $\widetilde{A}_{k}$-neurons being appropriate to $A$-neurons.

4.6. For each $\widetilde{A}_{k}$-neuron:

4.6.1) to determine an error of the output $E_{k}$;

4.6.2) to change the values of the vector of parameters $\mu_{\widetilde{A}_{k}}\left(p_{k}\right)$ by the formula (5).

4.7. To go to step 3 of the algorithm.

5. To determine the error of the model $E=\frac{r e s}{N}$.

6. If $E>0$, then go to step 2 of the algorithm and perform the training until error $E$ equals zero or changes.

As a result of learning a fuzzy neural network, a system of fuzzy-production rules with the identified parameter values is generated.

\section{Results and Discussion}

Oriented to the developed mathematical software, one has implemented a software package on the basis of which the studies on assessing the classifying ability of the fuzzy rules systems being formed using the example of analyzing certain data from the UCI Machine Learning Repository have been conducted [23]. Table 1 presents the characteristics of the data.

\begin{tabular}{|l|l|l|l|l|}
\hline The set of data & $\begin{array}{l}\text { The number of input } \\
\text { parameters }\end{array}$ & $\begin{array}{l}\text { The types of input } \\
\text { parameters }\end{array}$ & $\begin{array}{l}\text { The volume of } \\
\text { sampling }\end{array}$ & $\begin{array}{l}\text { The number of } \\
\text { classes }\end{array}$ \\
\hline Iris Plants Database & 4 & numerical & 150 & 3 \\
\hline BUPA Liver Disorders & 6 & numerical & 345 & 2 \\
\hline Australian Credit Approval & 14 & $\begin{array}{l}\text { numerical } \\
\text { categorial }\end{array}$ & 690 & 2 \\
\hline German Credit Data & 20 & $\begin{array}{l}\text { numerical, } \\
\text { categorial }\end{array}$ & 1000 & 2 \\
\hline
\end{tabular}

Table 1: The Characteristics of Source Data for Testing the Model 
The first set corresponds to the task of classifying Fisher irises. The second set presents the data for assessing the condition of the human liver. The third set describes the task of identifying shady bank card transactions. The fourth set describes the task of making a decision on allowance of a consumer credit.

Table 2 presents the results of the accuracy of assessing the state of objects based on the generated fuzzy rules. Fuzzy neural network learning was conducted both on the source data and on the data with missing values.

\begin{tabular}{|c|c|c|c|c|c|}
\hline The set of data $\%$ of absent data & 0 & 5 & 10 & 15 & 20 \\
\hline Iris Plants Database & 0,98 & 0,967 & 0,92 & 0,867 & 0,78 \\
\hline BUPA Liver Disorders & 0,762 & 0,751 & 0,719 & 0,667 & 0,574 \\
\hline Australian Credit Approval & 0,859 & 0,855 & 0,836 & 0,826 & 0,793 \\
\hline German Credit Data & 0,827 & 0,825 & 0,811 & 0,798 & 0,76 \\
\hline
\end{tabular}

Table 2: The Accuracy of the Classification of Different Sets of Data

The classifying ability of the generated fuzzy rules turned out to be comparable with the accuracy of other known methods (a team of neural networks, a multi-layer perceptron, a classifier on fuzzy logic) [24], surpasses the Bayes and Boosting methods [25] by $15 \%$, the co-evolutionary method of learning algorithmic compositions by $9 \%$ [26] and the method of fuzzy classification of objects with discrete output by 3\% [27]. However, in contrast to the considered methods, the absence of a part of the input data in the proposed approach does not make it impossible to execute an inference algorithm for assessing the state of an object. At the same time, with an increase in the volume of missing values, the classification accuracy decreases slightly. This proves the effectiveness of the proposed approach for assessing the state of an object in uncertainty, heterogeneity and the lack of a part of the analyzed data.

\section{Summary}

The described model enables to generate fuzzy rules for assessing the state of objects in uncertainty. The study of fuzzy rules obtained by analyzing certain data showed their high classifying ability, including when testing on samples with missing values. This indicates the high efficiency of the model of the fuzzy neural network and its suitability for generating fuzzy rules for assessing the state of objects in various subject areas.

\section{Conclusion}

Thus, the developed model of a fuzzy neural network is an effective tool for generating fuzzy rules for assessing the state of objects in uncertainty. In the future, in order to develop the proposed methods for generating fuzzy rules, it is advisable to improve the developed software, as well as to develop and to put into practice intelligent systems for assessing the state of objects in various fields [29].

\section{Acknowledgement}

The work is performed according to the Russian Government Program of Competitive Growth of Kazan Federal University. This work was supported by the Russian Federation Ministry of Education and Science, project № 8.6141.2017/8.9.

\section{References}

[1] Zhou D., Mao S., Cheng J., Cao X., Hu J. A Bayesian statistical analysis of stochastic phenotypic plasticity model of cancer cells // Journal of Theoretical Biology. - 2018. - No. - 454. - P. 70-79.

[2] Anikin I.V., Gazimov R.M. Privacy preserving DBSCAN clustering algorithm for vertically partitioned data in distributed systems // Proceedings of 2017 International Siberian Conference on Control and Communications, SIBCON 2017.

[3] Thilina A., Attanayake S., Samarakoon S., Edirisinghe T., Krishnadeva K. Intruder detection using deep learning and association rule mining // Proceedings of 16th IEEE International Conference on Computer and Information Technology, CIT 2016. - P. 615-620.

[4] Nikolaeva Z. Imitation model of solar radiation and concentrations of ozone and nitrogen dioxide in the atmosphere // Oxidation Communications. - 2018. - No. 41(2). - P. 231-238.

[5] Akhmetvaleev A.M., Katasev A.S. Neural network model of human intoxication functional state determining in some problems of transport safety solution // Computer Research and Modeling. - 2018. - No. 10 (3). - P. 285293.

[6] Ge C., Wang B., Wei X., Liu Y. Exponential synchronization of a class of neural networks with sampled-data control // Applied Mathematics and Computation. - 2017. - No. 315. - P. 150-161.

[7] Guskov G., Namestnikov A., Yarushkina N. Approach to the search for similar software projects based on the UML ontology // Advances in Intelligent Systems and Computing. - 2018. - No. 680. - P. 3-10.

[8] Katasev A.S., Kataseva D.V. Neural network diagnosis of anomalous network activity in telecommunication systems // Proceedings of IEEE Conference Dynamics of Systems, Mechanisms and Machines, Dynamics 2016.

[9] Nazemi A., Dehghan M. A neural network method for solving support vector classification problems // Neurocomputing. - 2015. - No. 152. - P. 369-376. 
[10] Ismagilov I.I., Khasanova S.F., Katasev A.S., Kataseva D.V. Neural network method of dynamic biometrics for detecting the substitution of computer // Journal of Advanced Research in Dynamical and Control Systems. 2018. - No. 10(10 Special Issue). - P. 1723-1728.

[11] Zadeh L.A. Fuzzy Sets // Information and Control. - 1965. - Vol. 8. - P. 338-353.

[12] Ismagilov, I.I., Khasanova, S.F. Short-term fuzzy forecasting of brent oil prices // Asian Social Science, 2015, 11 , pp. 60-67.

[13] Nazarov A.O., Anikin I.V. Clusterization of objects with fuzzy parameter's values // Proceedings of 11th IEEE Conference Dynamics of Systems, Mechanisms and Machines, Dynamics 2017.

[14] Salakhutdinov R.Z., Ismagilov I.I., Rubtsov A.V. A neural-fuzzy approach to economic data classification // International Conference on Fuzzy Sets and Soft Computing in Economics and Finance. Proceedings. Volume II, 2004. - P.394-400.

[15] Garaeva A., Makhmutova F., Anikin I., Sattler K.-U. A framework for co-location patterns mining in big spatial data // Proceedings of 2017 20th IEEE International Conference on Soft Computing and Measurements, SCM 2017.

[16] Liu X., Wang H., Gao C., Chen M. Adaptive fuzzy funnel control for a class of strict feedback nonlinear systems // Neurocomputing. - 2017. - No. 241. - P. 71-80.

[17] Namestnikov A.M., Filippov A.A., Avvakumova V.S. An ontology-based model of technical documentation fuzzy structuring // CEUR Workshop Proceedings. - 2016. - No. 1687. - P. 63-74.

[18] Vaskovic M., Kodogiannis V.S., Budimir D. An adaptive fuzzy logic system for the compensation of nonlinear distortion in wireless power amplifiers // Neural Computing and Applications. - 2018. - No. 30(8). - P. 25392554.

[19] Tron E., Margaliot M. Mathematical Modeling of Observed Natural Behavior: a Fuzzy Logic Approach // Fuzzy Sets and Systems. - 2004. - Vol. 146. - P. 437-450.

[20] Yarushkina N. Soft computing and complex system analysis // International Journal of General Systems. - 2001. - No. 30(1). - P. 71-88.

[21] Hassan N., Sayed O.R., Khalil A.M., Ghany M.A. Fuzzy Soft Expert System in Prediction of Coronary Artery Disease // International Journal of Fuzzy Systems. - 2017. - No. 19(5). - P. 1546-1559.

[22] Katasev A.S., Kataseva D.V., Emaletdinova L.Yu. Neuro-fuzzy model of complex objects approximation with discrete output // Proceedings of 2nd International Conference on Industrial Engineering, Applications and Manufacturing, ICIEAM 2016.

[23] Bache K., Lichman M. UCI Machine Learning Repository [http://archive.ics.uci.edu/ml]. Irvine, CA: University of California, School of Information and Computer Science. - 2013.

[24] Bukhtoyarov V.V. Three-Step Evolutionary Method of Forming the Teams of Neural Networks for Solving Classification Problems // Software Products and Systems.- 2012. - № 4. - P. 101-106.

[25] Schapire R. The boosting approach to machine learning: An overview // MSRI Workshop on Nonlinear Estimation and Classification, Berkeley, CA, 2001. - 23 p.

[26] Vorontsov K.V., Kanevsky D.Y. Co-Evolutionary Method of Teaching Algorithmic Compositions // Tavricheskii Vestnik of Informatics and Mathematics. - 2005. - № 2. - P. 51-66.

[27] Shtovba S. D. Identification of Non-Linear Dependencies By Means of Fuzzy Logical Inference in the System of MATLAB // Math in Applications. - 2003. - № 2(2). - P. 9-15.

[28] Katasev A.S., Kataseva D.V. Expert diagnostic system of water pipes gusts in reservoir pressure maintenance processes // 2nd International Conference on Industrial Engineering, Applications and Manufacturing, ICIEAM 2016 - Proceedings, 7911651. 